\title{
BOUNDS ON STOP-LOSS PREMIUMS FOR COMPOUND DISTRIBUTIONS
}

\author{
BY R. KAAS \\ University of Amsterdam \\ AND \\ M. J. Goovaerts \\ University of Amsterdam and K. U. Leuven
}

\begin{abstract}
Upper and lower bounds are derived for the stop-loss premium of compound distributions with fixed claim number distribution and known mean, variance and range for the claim severity distribution.
\end{abstract}

\section{INTRODUCTION}

In this paper we investigate bounds on stop-loss premiums for compound distributions

$$
S=X_{1}+X_{2}+\cdots+X_{N}
$$

where the claim number distribution $F_{N}$ is fixed (e.g., Poisson $(\lambda)$ ) and where the claim severity distribution $F_{X}$ is restricted to have

$$
\begin{gathered}
F_{x}(-0)=0 \\
F_{X}(b)=1 \\
E[X]=\mu
\end{gathered}
$$

for some finite $b$ and $\mu \in[0, b]$.

The stop-loss premium of $S$ with stop-loss point $t$ will be denoted by

$$
\pi\left(t ; F_{X}\right)=\int_{t}^{\infty}(x-t) d\left[\sum_{n=0}^{\infty} \operatorname{Pr}(N=n) F_{X}^{n^{*}}(x)\right]
$$

BÜHLMANN et al. (1977), introducing the concept of stop-loss ordering, derived bounds for $\pi\left(t ; F_{X}\right)$.

In fact, when the random variable $X^{-}$equals $\mu$ with probability one, and $X^{+}$ has range $\{0, b\}$ and mean $\mu$,

$$
\pi\left(t ; F_{X^{-}}\right) \leqslant \pi\left(t ; F_{X}\right) \leqslant \pi\left(t ; F_{X^{+}}\right)
$$

uniformly in $t$ and for all $X$ satisfying (2).

ASTIN BULLETIN Vol 16 , No. 1 
To prove that this is true first observe that $\alpha$ and $\beta$ exist such that

$$
\begin{array}{ll}
F_{X^{-}}(x) \leqslant F_{X}(x) & \text { for } x<\alpha \\
F_{X^{-}}(x) \geqslant F_{X}(x) & \text { for } x \geqslant \alpha \\
F_{X^{+}}(x) \leqslant F_{X}(x) & \text { for } x \geqslant \beta \\
F_{X^{+}}(x) \geqslant F_{X}(x) & \text { for } x<\beta .
\end{array}
$$

As $E\left[X^{-}\right]=E[X]=E\left[X^{+}\right]$, this means that $X^{-}$is less dangerous than $X$ and $X^{+}$is more dangerous. As a more dangerous distribution has higher stop-loss premiums, we have

$$
X^{-} \propto X \propto X^{+}
$$

where $\propto$ denotes stop-loss order. Stop-loss order is preserved under compounding, so

$$
\sum_{i=1}^{N} X_{i}^{-} \propto \sum_{i=1}^{N} X_{i} \propto \sum_{i=1}^{N} X_{i}^{+}
$$

which is equivalent to (4) holding for all real $t$. For a more detailed proof, see GoOvAERTs et al. (1984).

Since $X^{-}$and $X^{+}$satisfy all requirements for $X$, the bounds in (4) are best possible, and $X^{-}$and $X^{+}$are extremal distributions.

It is not possible to give such extreme distributions when the variance of $X$ is also fixed, say

$$
\operatorname{Var}(X)=\sigma^{2} .
$$

With the techniques of GoovAERTs et al. (1984) one may compute extreme values of stop-loss premiums, but unfortunately the corresponding distributions depend on the value of the stop-loss point chosen. There is no severity distribution in this class that is smallest or largest in the sense of stop-loss order.

In Section 2 we exhibit random variables $Z^{-}$and $Z^{+}$that give bounds like (4), uniformly in $t$. These bounds are not the best possible, since $Z^{-}$and $Z^{+}$ have variances different from $\sigma^{2}$. They are, however, the greatest lower and least upper bound with respect to dangerousness.

In Section 3 we give a numerical illustration using the examples of GERBER (1982).

\section{ANALYTICAL BOUNDS ON DISTRIBUTION FUNCTIONS}

In GoovaerTs and KAAs (1986) extreme values are given for distributions $F_{X}$ with range $[0, b]$ and the first few moments fixed. When $X$ has mean $\mu$ and variance $\sigma^{2}$, we have

$$
F^{\prime}(x) \leqslant F_{X}(x) \leqslant F^{u}(x)
$$

with the values of $F^{l}$ and $F^{u}$ given in the following table, where $z=(x-\mu) / \sigma$ and $d=b \mu-\mu^{2}-\sigma^{2} \geqslant 0$ are used for notational convenience. 
TABLE 1

BOUNDS FOR Distribution FUNCTIONS WITH RANGE $[0, b]$, MEAN $\mu$ AND VARIANCE $\sigma^{2}$

\begin{tabular}{ccc}
\hline$x:$ & $F^{l}(x)$ & $F^{u}(x)$ \\
\hline $0<x \leqslant \frac{d}{b-\mu}$ & 0 & $\frac{1}{1+z^{2}}$ \\
$\frac{d}{b-\mu} \leqslant x \leqslant b-\frac{d}{\mu}$ & $1-\frac{\mu}{b}-\frac{d}{b x}$ & $1-\frac{\mu}{b}+\frac{d}{b(b-x)}$ \\
$b-\frac{d}{\mu} \leqslant x \leqslant b$ & $1-\frac{1}{1+z^{2}}$ & 1 \\
\hline
\end{tabular}

Now define the following two severity distributions:

$$
F_{z^{+}}(x)= \begin{cases}F^{u}(x) & 0 \leqslant x \leqslant \alpha \\ F^{u}(\alpha) & \alpha \leqslant x \leqslant \beta \\ F^{l}(x) & \beta \leqslant x \leqslant b\end{cases}
$$

where $\alpha$ and $\beta$ satisfy

$$
\begin{aligned}
\alpha & =\mu+\frac{\sigma}{\sigma^{2}+\mu(b-\mu)} \\
& \times\left[\sigma(b-2 \mu)-\sqrt{\sigma^{2}(b-2 \mu)^{2}+\left(\sigma^{2}+\mu(b-\mu)\right)^{2}}\right] \\
\beta & =\mu+\frac{\sigma}{\sigma^{2}+\mu(b-\mu)} \\
& \times\left[\sigma(b-2 \mu)+\sqrt{\sigma^{2}(b-2 \mu)^{2}+\left(\sigma^{2}+\mu(b-\mu)\right)^{2}}\right]
\end{aligned}
$$

and

$$
F_{Z^{-}}(x)=\left\{\begin{array}{ll}
F^{l}(x) & 0 \leqslant x<\mu \\
F^{u}(x) & \mu \leqslant x \leqslant b
\end{array} .\right.
$$

With $d$ as in table 1 , we have $\alpha \leqslant d /(b-\mu)$ and $\beta \geqslant b-d / \mu$. To check that $F_{Z^{+}}$ is well-defined and

$$
E\left[Z^{-}\right]=E\left[Z^{+}\right]=\mu
$$

is a laborious process but involves only elementary calculus. Since the distribution $G$ with $d G(\alpha)=F^{u}(\alpha)=1-d G(\beta)$ has mean $\mu$ and variance $\sigma^{2}$, we have $\operatorname{Var}\left(Z^{+}\right)>\sigma^{2}$.

In fact, it may be shown that, writing $t^{*}=(t-\mu) / \sigma$ for all $t$,

$$
\operatorname{Var}\left(Z^{+}\right)=\sigma^{2}\left(1+\ln \left(\frac{\left(1+b^{* 2}\right)\left(1+0^{* 2}\right)}{\left(1+\alpha^{* 2}\right)\left(1+\beta^{* 2}\right)}\right)\right)
$$

In the same way, considering the distribution $H$ with

$$
d H(0)=F^{l}(\mu), \quad d H(\mu)=F^{u}(\mu)-F^{l}(\mu), \quad d H(b)=1-F^{u}(\mu),
$$


which has mean $\mu$ and variance $\sigma^{2}$, one shows that $\operatorname{Var}\left(Z^{-}\right)<\sigma^{2}$. Because of (10) we have immediately that $Z^{-}$is less dangerous than any $X$, and $Z^{+}$is more dangerous, so

$$
\pi\left(t ; Z^{-}\right) \leqslant \pi(t ; X) \leqslant \pi\left(t ; Z^{+}\right)
$$

uniformly for all $t$ and for all feasible $X$.

Now let $W$ be a random variable with $d F_{W}(x)>0$ for some $x$ where also $F^{l}(x)<F_{W}(x)<F^{u}(x)$. It is easy to construct a feasible $X$ with $F_{X}(x)=F_{W}(x)$ and $x$ outside the spectrum of $X: d F_{X}(x)=0$. But then either $X$ is more dangerous than $W$, or $X$ and $W$ are not comparable because $F_{X}$ and $F_{W}$ have two more more sign changes. So to be more dangerous than all $X, F_{W}$ must be first above $F^{u}$, then constant between $F^{u}$ and $F^{l}$, then below $F^{l}$. But then it is easy to see that $F_{Z^{+}}$and $F_{W}$ have only one point of intersection, so $Z^{+}$is less dangerous than $W$.

Reasoning along the same lines for $Z^{-}$we may conclude that among the distributions more dangerous than any feasible $X, Z^{+}$is the least dangerous, whereas $Z^{-}$is the most dangerous less dangerous distribution. In this sense $Z^{+}$ and $Z^{-}$are optimal choices.

\section{NUMERICAL ILLUSTRATION}

In order to assess the quality of the bounds derived in the previous section, we give a numerical example. In Gerber (1982) methods are described to bound as well as to approximate stop-loss premiums of compound Poisson distributions. His method to obtain a lower bound using mass concentration does not always give an arithmetic discrete distribution, so we used the method of matching (two) moments, which is much more exact with the same computational effort. To obtain Gerber's uniform $(1,3)$ claim severity distribution as a special case, we took $b=3, \mu=2$ and $\sigma^{2}=\frac{1}{3}$ in our examples, the claim numbers being Poisson $(\lambda)$ with $\lambda=1,10$ and 100 .

TABLE 2

Bounds for Stop-loss Premiums with Claim-Range [0,3], Mean 2, Variance $\frac{1}{3}$ and Claim Number Poisson (1)

\begin{tabular}{clcccc}
\hline \hline $\begin{array}{c}\text { Stop-Loss } \\
\text { Point } t\end{array}$ & $\begin{array}{c}\text { Gerber's } \\
\text { Exact Value }\end{array}$ & $\begin{array}{c}\text { Upper } \\
\text { Bound (4) }\end{array}$ & $\begin{array}{c}\text { Upper } \\
\text { Bound (15) }\end{array}$ & $\begin{array}{c}\text { Lower } \\
\text { Bound (15) }\end{array}$ & $\begin{array}{c}\text { Lower } \\
\text { Bound (4) }\end{array}$ \\
\hline 0 & 2.000 & $100.0 \%$ & $100.0 \%$ & $100.0 \%$ & $100.0 \%$ \\
2 & $8.277 \times 10^{-1}$ & 124.1 & 113.5 & 89.3 & 88.9 \\
4 & $2.689 \times 10^{-1}$ & 147.2 & 124.2 & 78.4 & 77.1 \\
6 & $7.184 \times 10^{-2}$ & 149.4 & 134.8 & 67.3 & 65.0 \\
8 & $1.627 \times 10^{-2}$ & 288.4 & 182.9 & 56.7 & 53.5 \\
10 & $3.254 \times 10^{-3}$ & 364.0 & 227.3 & 46.2 & 42.3 \\
12 & $5.815 \times 10^{-4}$ & 365.6 & 261.1 & 36.7 & 32.6 \\
14 & $9.346 \times 10^{-5}$ & 921.3 & 356.9 & 28.7 & 24.6 \\
16 & $1.366 \times 10^{-5}$ & 1166.9 & 495.0 & 22.1 & 18.3 \\
18 & $1.840 \times 10^{-6}$ & 1154.0 & 597.4 & 16.8 & 13.3 \\
20 & $2.302 \times 10^{-7}$ & 3578.6 & 807.5 & 12.5 & 9.5 \\
\hline
\end{tabular}


TABLE 3

Bounds for Stop-loss Premiums with Claim Range [0,3], Mean 2, VARiance $\frac{1}{3}$ and Claim Number Poisson (10)

\begin{tabular}{clcccc}
\hline \hline $\begin{array}{c}\text { Stop-Loss } \\
\text { Point } t\end{array}$ & $\begin{array}{c}\text { Gerber's } \\
\text { Exact Value }\end{array}$ & $\begin{array}{c}\text { Upper } \\
\text { Bound (4) }\end{array}$ & $\begin{array}{c}\text { Upper } \\
\text { Bound (15) }\end{array}$ & $\begin{array}{c}\text { Lower } \\
\text { Bound (15) }\end{array}$ & $\begin{array}{c}\text { Lower } \\
\text { Bound (4) }\end{array}$ \\
\hline 15 & 5.757 & $105.4 \%$ & $103.0 \%$ & $99.0 \%$ & $99.0 \%$ \\
20 & 2.626 & 118.2 & 109.2 & 95.6 & 95.3 \\
25 & $9.321 \times 10^{-1}$ & 141.6 & 121.2 & 91.6 & 91.6 \\
30 & $2.563 \times 10^{-1}$ & 175.9 & 141.1 & 81.9 & 80.7 \\
35 & $5.507 \times 10^{-2}$ & 257.4 & 172.0 & 74.7 & 74.7 \\
40 & $9.383 \times 10^{-3}$ & 380.3 & 218.8 & 61.2 & 59.2 \\
45 & $1.289 \times 10^{-3}$ & 551.5 & 289.4 & 53.1 & 53.1 \\
50 & $1.449 \times 10^{-4}$ & 1028.0 & 396.5 & 40.2 & 37.9 \\
55 & $1.355 \times 10^{-5}$ & 1762.1 & 560.8 & 33.3 & 33.3 \\
60 & $1.067 \times 10^{-6}$ & 2829.9 & 816.8 & 23.5 & 21.5 \\
65 & $7.175 \times 10^{-8}$ & 6465.7 & 1226.1 & 18.6 & 18.6 \\
\hline
\end{tabular}

TABLE 4

Bounds for Stop-loss Premiums with Claim Range [0,3], Mean 2, Variance $\frac{1}{3}$ and Claim Number Poisson (100)

\begin{tabular}{clcccc}
\hline \hline $\begin{array}{c}\text { Stop-Loss } \\
\text { Point } t\end{array}$ & $\begin{array}{c}\text { Gerber's } \\
\text { Exact Value }\end{array}$ & $\begin{array}{c}\text { Upper } \\
\text { Bound (4) }\end{array}$ & $\begin{array}{c}\text { Upper } \\
\text { Bound (15) }\end{array}$ & $\begin{array}{c}\text { Lower } \\
\text { Bound (15) }\end{array}$ & $\begin{array}{c}\text { Lower } \\
\text { Bound (4) }\end{array}$ \\
\hline 180 & $2.177 \times 10^{-1}$ & $104.4 \%$ & $102.2 \%$ & $99.1 \%$ & $99.1 \%$ \\
200 & 8.304 & 117.7 & 109.2 & 96.1 & 96.0 \\
220 & 1.959 & 152.0 & 126.4 & 89.2 & 88.9 \\
240 & $2.647 \times 10^{-1}$ & 230.0 & 161.6 & 78.1 & 77.6 \\
260 & $1.992 \times 10^{-2}$ & 420.9 & 229.6 & 64.2 & 63.5 \\
280 & $8.395 \times 10^{-4}$ & 922.3 & 364.7 & 49.3 & 48.4 \\
300 & $2.319 \times 10^{-5}$ & 2846.4 & 725.9 & 33.8 & 33.4 \\
\hline
\end{tabular}

\section{REFERENCES}

Bühlmann, H., Gagliardi, B., Gerber, H. and Straub, E. (1977) Some Inequalities for Stop-Loss Premiums, ASTIN-Bulletin IX, 75-83.

Gerber, H. U. (1982) On the Numerical Evaluation of the Distribution of Aggregate Claims and its Stop-Loss Premiums. Insurance: Mathematics and Economics I, 13-18.

GoovaERTS, M. J. and KaAs, R. (1986) Analytical Bounds on Distributions Under Integral Constraints, to be published.

Goovaerts, M. J., De Vylder, F. and Haezendonck, J. (1984) Insurance Premiums. NorthHolland Publishing Company: Amsterdam.

R. KaAs and M. J. Goovaerts

Universiteit van Amsterdam, Jodenbreestraat 23, NL-1011 WH Amsterdam, The Netherlands. 